\title{
THE EDITORIAL PAGE
}

In 1962 the American Meteorological Society launched the Journal of Applied MeteOROLOGY as . . " a medium for the publication of research concerned with the application of the atmospheric sciences to operational and practical goals." Now, in its eighth year, this objective of the Journal continues to be the primary guideline for its fourth Editor, just as it was for the first three. The materials printed in JAM shall continue to meet the tests of research, application, and operational and practical goals, reinforced, of course, by sound professional judgement on the scientific and technical merits of the research reported.

However, even though this objective of the JouRnaL is firmly in place, there are many subsidiary considerations which do not derive automatically from it. What is a reasonable time between receipt and publication of a manuscript? How are "sound professional judgements" to be assured? Are anonymous reviews the best way to get these judgements? What is an appropriate balance of papers among the myriad specialties which have developed in the fields of meteorology and its allied sciences? Should applications of meteorological research to relevant social problems be confined to reporting the meteorological side of the problem in JAM?

Providing the answers to these and other questions is, of course, the responsibility of the Editor of the Jourval. However, in the belief that an open discussion of these questions will help to make the JOURNAI a better medium of communication, we are initiating with this issue a new feature, the Editorial Page. From time to time we will present here information and discussions on the mechanics and philosophy of JAM. To do so is to invite "Letters to the Editor," and we do invite them, as guides to editorial policy and, hopefully, a constructive, dynamic, and progressive program of improvement of the scientific communications to which the Journal or Applied Meteorology is dedicated.

Glenn R. Hilst, EDITOR 